\title{
A FINITE DIMENSIONAL LINEAR PROGRAMMING APPROXIMATION OF MATHER'S VARIATIONAL PROBLEM
}

\author{
LUCA GRANIERI ${ }^{1}$
}

\begin{abstract}
We provide an approximation of Mather variational problem by finite dimensional minimization problems in the framework of $\Gamma$-convergence. By a linear programming interpretation as done in [9] we state a duality theorem for the Mather problem, as well a finite dimensional approximation for the dual problem.
\end{abstract}

Mathematics Subject Classification. 37J50, 49Q20, 49N60, 74P20, 65K10.

Received December 18, 2008.

Published online October 9, 2009.

\section{INTRODUCTION}

Mather variational principle, introduced by Mather in [18,19], is an important tool in Lagrangian dynamic. In recent years, several authors have studied this topic in connections with various fields such as weak Kam Theory [5,8,10,11], Monge-Kantorovich mass transportation and geometric measure theory [3,4,6,13,14]. In this paper we discuss a finite dimensional approximation of Mather variational problem on the flat torus $\mathbb{T}^{N}$, following a linear programming interpretation of Mather's variational principle outlined in [9].

\subsection{Mather's problem}

We will consider a compact Riemannian manifold $(M, g)$ without boundary and a Lagrangian $L: T M \rightarrow \mathbb{R}$ which satisfies the following properties:

(1) $L$ is regular (at least $\mathcal{C}^{2}$ );

(2) $L(x, \cdot)$ is superlinear in the fiber uniformly on $x \in M$;

(3) $L(x, \cdot)$ is strictly convex in the fiber for all $x \in M$.

The regularity requirement on $L$ is crucial to consider regular solutions of the Hamilton-Jacobi equation (see Sect. 3.1).

A measure $\mu \in \mathcal{M}(T M)$ is said to be closed if for all exact forms $\omega$ it results

$$
\int_{T M}\langle\omega(x), v\rangle \mathrm{d} \mu=0
$$

Keywords and phrases. Mather problem, minimal measures, linear programming, $\Gamma$-convergence.

1 Dipartimento di Matematica Politecnico di Bari, via Orabona 4, 70125 Bari, Italy. 1.granieri@poliba.it, granieriluca@libero.it 
According to this definition we set

$$
\mathcal{M}^{c}=\{\mu \in \mathcal{M}(T M) \mid \mu \text { is closed, with compact support, } \mu \in \mathcal{P}(T M)\},
$$

where $\mathcal{P}(T M)$ stands for probability measures, i.e. $\mu \geq 0$ and $\mu(T M)=1$. To each measure $\mu \in \mathcal{M}^{c}$ we can associate the homology class of $\mu$ which we will denote by $[\mu] \in H_{1}\left(M, \mathbb{R}\right.$ ) (by duality with $H^{1}$ ). Indeed, $\mu$ acts in a natural way on the set of the closed 1-forms on $M$ by

$$
\omega \mapsto \int_{T M}\langle\omega(x), v\rangle \mathrm{d} \mu
$$

and thanks to condition (1.1) this action passes to the quotient by the exact forms.

Once we fix an homology class $[h]$, Mather's variational problem amounts to:

$$
\min _{\mathcal{M}^{c}}\left\{\int_{T M} L(x, v) \mathrm{d} \mu \mid[\mu]=[h]\right\} .
$$

We also define the action of $\mu$ by

$$
\mathcal{A}(\mu):=\int_{T M} L(x, v) \mathrm{d} \mu .
$$

A remarkable property of problem (1.3) is the following: we minimize an action functional which depends on $L$ on measures which are merely closed. However, it turns out that the minimal measures are also invariant for the flow associated to the Lagrangian $L$, see for example $[3,5,11,17]$.

Consider the $N$-dimensional flat torus $\mathbb{T}^{N}$. In this case it is known that the homology $H_{1}\left(\mathbb{T}^{N}, \mathbb{R}\right)$ and the cohomology $H^{1}\left(\mathbb{T}^{N}, \mathbb{R}\right)$ are $N$-dimensional vector spaces. Moreover, the cohomology on $\mathbb{T}^{N}$ is generated by the classes $\left[d x_{1}\right],\left[d x_{2}\right], \ldots,\left[d x_{N}\right]$. In other words, for every closed 1 -forms $\omega$ on $\mathbb{T}^{N}$ there exists $P \in \mathbb{R}^{N}$ and $f \in \mathcal{C}^{1}\left(\mathbb{T}^{N}\right)$ such that $\omega=P+d f$. For details we refer for instance to [15]. Fixed $Q \in \mathbb{R}^{N}$, denoted by $\mathcal{M}_{c}$ the space of measures having compact support, in this setting Mather's problem can be formulated as follows:

$$
\operatorname{Minimize}\left\{\mathcal{A}(\mu) \mid \mu \in \mathcal{M}_{c}\left(\mathbb{T}^{N} \times \mathbb{R}^{N}\right)\right\}
$$

under the following constraints:

(1) $\mu \in \mathcal{P}\left(\mathbb{T}^{N} \times \mathbb{R}^{N}\right)$;

(2) $\forall f \in \mathcal{C}^{1}\left(\mathbb{T}^{N}\right): \int_{\mathbb{T}^{N} \times \mathbb{R}^{N}}\langle d f, v\rangle \mathrm{d} \mu=0$;

(3) $\int_{\mathbb{T}^{N} \times \mathbb{R}^{N}} v \mathrm{~d} \mu=Q$.

Observe that the second constraint in the above list correspond to the closeness of $\mu$ while the third one is equivalent to fix the homology class $[\mu]$. Therefore, Mather's problem corresponds to minimize the functional $\mathbb{P}$ defined as

$$
\mu \in \mathcal{M}_{c}\left(\mathbb{T}^{N} \times \mathbb{R}^{N}\right) \mapsto \mathbb{P}(\mu)=\mathcal{A}(\mu)+I_{K}(\mu),
$$

where $I_{K}$ is the indicator function of the set $K$ of measures which satisfy the above constraints.

\subsection{Linear programming problems}

A finite dimensional linear programming problem is a minimizing problem of the following form

$$
\left\{\begin{array}{l}
\min \langle c, x\rangle \\
x \geq 0 \\
A x=b
\end{array}\right.
$$


for $c \in \mathbb{R}^{N}, b \in \mathbb{R}^{M}$ and $A$ a $N \times M$ matrix. Here we write $x \geq 0$ to mean $x_{i} \geq 0$ for every $i=1, \ldots, N$. We can associate to problem (1.5) a dual problem in the following canonical way

$$
\left\{\begin{array}{l}
\max \langle b, y\rangle \\
A^{t} y \leq c
\end{array}\right.
$$

where $A^{t}$ denotes the transpose matrix of $A$. Observe that in the dual problem (1.6) we do not ask for positive solutions. Denoting respectively by $K, H$ the set of constraints of problem (1.5) and (1.6) one has

$$
\forall x \in K, \forall y \in H: \quad\langle b, y\rangle \leq\langle c, x\rangle .
$$

Therefore, if we denote by $\mathcal{P}$ the minimum value of problem (1.5) and by $\mathcal{D}$ the maximum value of problem (1.6) we have the following comparison

$$
\mathcal{D} \leq \mathcal{P} .
$$

We have the equality sign in the above inequality if the following complementary slackness condition holds.

Lemma 1.1 (slackness condition). Let $x_{0} \in K$ and $y_{0} \in H$ such that

$$
\left\langle b, y_{0}\right\rangle=\left\langle c, x_{0}\right\rangle .
$$

Then $x_{0}$ is a solution of problem (1.5), while $y_{0}$ is a solution of problem (1.6). Moreover it results $\mathcal{P}=\mathcal{D}$.

A remarkable fact in finite dimensional linear programming is the following duality theorem:

Theorem 1.1 (strong duality). If one among the problems (1.5) and (1.6) admits a solution then also the other problem admits a solution. Moreover, it results $\mathcal{P}=\mathcal{D}$.

As references for linear programming problems we refer for instance to [2,21]. An infinite dimensional linear programming problem could be stated for real topological vector spaces $X, Y$. We assume $X$ endowed with a partial ordering, compatible with the linear structure, which induces a partial ordering on the dual space $X^{*}$. Given $c^{*} \in X^{*}, b \in Y$ and $A: X \rightarrow Y$ a continuous linear operator, denoting by $\langle$,$\rangle the pairing between a space$ and its dual, a linear programming problem could be stated as follows

$$
\left\{\begin{array}{l}
\min \left\langle c^{*}, x\right\rangle \\
x \geq 0 \\
A x=b .
\end{array}\right.
$$

Denoting by $A^{*}: Y^{*} \rightarrow X^{*}$ the adjoint operator of $A$, the dual problem of (1.9) amounts to

$$
\left\{\begin{array}{l}
\max \left\langle y^{*}, b\right\rangle \\
A^{*} y^{*} \leq c^{*}
\end{array}\right.
$$

In general, in this setting a strong duality assertion like Theorem 1.1 does not hold. However, the slackness condition remains true. Denoting by $K, H$ the set of constraints of problem (1.9) and (1.10) respectively, we have the following:

Theorem 1.2 (weak duality). For every $x \in K, y^{*} \in H$ it results

$$
\left\langle y^{*}, b\right\rangle \leq\left\langle c^{*}, x\right\rangle \cdot
$$

Moreover, if for some $x_{0} \in K, y_{0}^{*} \in H$ it results

$$
\left\langle y_{0}^{*}, b\right\rangle=\left\langle c^{*}, x_{0}\right\rangle,
$$

then $x_{0}$ is a solution of problem (1.9), while $y_{0}^{*}$ is a solution of problem (1.10). 


\subsection{Description of the results}

In the paper [9] the authors outline a linear programming formulation of Mather problem. In a large heuristic way they discuss some formal implications of linear programming duality to the Mather theory. Following these guide-lines, in this paper we provide an approximation of Mather problem by finite dimensional minimization problems. We perform this approximation in the framework of $\Gamma$-convergence. In particular, discretizing the phase-space $\mathbb{T}^{N} \times \mathbb{R}^{N}$, we find a sequence of finite dimensional linear programming problems $\mathbb{P}_{n}$ which $\Gamma$-converge to the Mather problem. Therefore we can recover a Mather minimizing measure by solving finite dimensional linear programming problems (see Thm. 2.2). As a consequence of recent developments of Hamilton-Jacobi and Mather theory [5,9-11,17,19] we state a duality result for Mather problem (see Sect. 3.1). Moreover, the finite dimensional duality provides also an approximation result for the dual problem (see Thm. 3.2).

\section{Discrete approximation of Mather problem}

Following Mather we restrict to consider measures with compact support. To pursue variational arguments, one need a notion of convergence which preserves the constraints. Therefore we get the following:

Definition 2.1. Let $\mu_{n}, \mu \in \mathcal{M}(T M)$. We say that $\mu_{n} \rightarrow \mu$ if $\mu_{n} \stackrel{*}{\rightarrow} \mu$ and $\int_{T M}|v| \mathrm{d} \mu_{n} \rightarrow \int_{T M}|v| \mathrm{d} \mu$ as $n \rightarrow+\infty$.

Given a continuous function $f: T M \rightarrow \mathbb{R}$ we say that $f$ grows at most linearly if there exist two constant $A, B \geq 0$ such that for every $(x, v) \in T M$ it results $|f(x, v)| \leq A+B|v|$. For the reader convenience we state the following (see also [17]):

Lemma 2.1. Let $\mu_{n}, \mu \in \mathcal{P}_{c}(T M)$ be probability measures having compact support. If $\mu_{n} \rightarrow \mu$ then

$$
\lim _{n \rightarrow+\infty} \int_{T M} f \mathrm{~d} \mu_{n}=\int_{T M} f \mathrm{~d} \mu
$$

for every continuous function which grows at most linearly.

Proof. Fix a large compact set $\mathcal{K}$ such that $\operatorname{supp}(\mu) \subset \mathcal{K}$ and $\mu(\partial \mathcal{K})=0$. Given a continuous functions $f$ which grows at most linearly we set $\varphi(v)=A+B|v|$. We evaluate

$$
\begin{aligned}
\left|\int_{T M} f \mathrm{~d}\left(\mu-\mu_{n}\right)\right| \leq & \left|\int_{\mathcal{K}} f \mathrm{~d}\left(\mu-\mu_{n}\right)\right|+\left|\int_{\mathcal{K}^{c}} f \mathrm{~d}\left(\mu-\mu_{n}\right)\right| \\
& \leq\left|\int_{\mathcal{K}} f \mathrm{~d}\left(\mu-\mu_{n}\right)\right|+\int_{\mathcal{K}^{c}}|f| \mathrm{d} \mu_{n} \leq\left|\int_{\mathcal{K}} f \mathrm{~d}\left(\mu-\mu_{n}\right)\right|+\int_{\mathcal{K}^{c}} \varphi(v) \mathrm{d} \mu_{n} \\
& =\left|\int_{\mathcal{K}} f \mathrm{~d}\left(\mu-\mu_{n}\right)\right|+\int_{T M} \varphi(v) \mathrm{d} \mu_{n}-\int_{\mathcal{K}} \varphi(v) \mathrm{d} \mu_{n}-\left(\int_{T M} \varphi(v) \mathrm{d} \mu-\int_{\mathcal{K}} \varphi(v) \mathrm{d} \mu\right) .
\end{aligned}
$$

Observe that in the above computation we have used the condition $\operatorname{supp}(\mu) \subset \mathcal{K}$ to infer

$$
\left|\int_{\mathcal{K}^{c}} f \mathrm{~d}\left(\mu-\mu_{n}\right)\right|=\left|\int_{\mathcal{K}^{c}} f \mathrm{~d} \mu_{n}\right| ; \quad \int_{T M} \varphi(v) \mathrm{d} \mu-\int_{\mathcal{K}} \varphi(v) \mathrm{d} \mu=\int_{\mathcal{K}^{c}} \varphi(v) \mathrm{d} \mu=0
$$

respectively in the second and the third line. We can now estimate

$$
\left|\int_{T M} f \mathrm{~d}\left(\mu-\mu_{n}\right)\right| \leq\left|\int_{\mathcal{K}} f \mathrm{~d}\left(\mu-\mu_{n}\right)\right|+\int_{\mathcal{K}} \varphi \mathrm{d}\left(\mu-\mu_{n}\right)+\int_{T M} \varphi \mathrm{d}\left(\mu_{n}-\mu\right) .
$$


By standard properties of weak-star convergence (see for example Prop. 1.62 of [1]) we get

$$
\lim _{n \rightarrow+\infty} \int_{\mathcal{K}} f \mathrm{~d}\left(\mu-\mu_{n}\right)=0=\lim _{n \rightarrow+\infty} \int_{\mathcal{K}} \varphi \mathrm{d}\left(\mu-\mu_{n}\right) .
$$

Moreover, since we are dealing with probability measures, $\mu_{n} \rightarrow \mu \Rightarrow \int_{T M} \varphi(v) \mathrm{d} \mu_{n} \rightarrow \int_{T M} \varphi(v) \mathrm{d} \mu$ as $n \rightarrow+\infty$. Therefore, by (2.1) the statement follows.

To realize a discrete approximation, we will allow to a measure $\mu$ to have an atom of negative mass at the origin. If the measure $\mu$ is not positive we consider the total variation $|\mu|$ given by $|\mu|=\mu^{+}+\mu^{-}$, where $\mu^{+}$and $\mu^{-}$denote the positive and negative parts corresponding to the Hahn decomposition of $\mu$. Moreover, since Mather problem deals with probability measures, by adding a constant to the Lagrangian $L(x, v)$ we may assume $L(0,0)=0$ without changing the minimizing problem. Therefore, throughout the rest of this paper $M=\mathbb{T}^{N}$ and $L(0,0)=0$.

\subsection{Formulation of the discrete functional $\mathbb{P}_{\boldsymbol{n}}$}

Let $n \geq 1$ be the discretization step. We consider the following discretization of $\mathbb{T}^{N}$

$$
I=\left\{\left(i_{1}, \ldots, i_{N}\right) \mid i_{h} \in\left\{0, \frac{1}{n}, \ldots, \frac{n-2}{n}, \frac{n-1}{n}\right\}\right\} .
$$

The number of elements of $I$ (which is $n^{N}$ ) will be denoted by $|I|$. The discretization of $\mathbb{R}^{N}$ is given by

$$
J=\left\{\left(j_{1}, \ldots, j_{N}\right) \mid j_{h} \in\left\{-n, \ldots,-\frac{1}{n}, 0, \frac{1}{n}, \ldots, n\right\}\right\} .
$$

We define the set $C$ as the set of atomic measures $\mu=\sum_{i, j} \mu_{i, j} \delta_{i, j} \in \mathcal{M}_{c}(T M)$ which satisfy the constraints:

$$
\left\{\begin{array}{l}
\mu_{i, j} \geq 0,(i, j) \neq(0,0), \\
\sum_{i, j} \mu_{i, j}=1, \\
\sum_{i, j} j \mu_{i, j}=Q, \\
\forall i \in I: \sum_{j} \sum_{h=1}^{N} j_{h}\left(\mu_{i-e_{h / n}, j}-\mu_{i, j}\right)=0 .
\end{array}\right.
$$

We define the functional $\mathbb{P}_{n}$ on $\mathcal{M}_{c}(T M)$ as

$$
\mathbb{P}_{n}(\mu)=\int_{T M} L(x, v) \mathrm{d} \mu+\Phi_{n}(\mu)+I_{C}(\mu),
$$

where $I_{C}(\mu)$ is the indicator function of the set $C$, while

$$
\Phi_{n}(\mu)=O(n) \mu_{0,0}^{-}+\sum_{i,|j|=n}|j| \mu_{i, j}-\sum_{i,|j|=n} L(i, j) \mu_{i, j},
$$

where $|j|$ denotes the norm, for instance the maximum norm, of the vector $j, O(n)$ is a strictly positive function such that $\lim _{n \rightarrow+\infty} O(n)=+\infty$ and $\lim _{n \rightarrow+\infty} \frac{O(n)}{n}=0$, while $\mu_{i, j}^{-}=\max \left(-\mu_{i, j}, 0\right)$. The introduction of the penalization term $\Phi_{n}(\mu)$ is motivated by the construction of discrete measures which approximate a given Mather's measure $\mu$ performed in Lemma 2.4. Indeed, to realize this approximation, a crucial step is to consider discrete measures which satisfy all the constraints (2.2). If such discrete measures do not satisfy all the constraints, we get a modified sequence of measures by adding suitable terms at the origin and on the points of the discretization such that $|j|=n$. The term $\Phi_{n}$, see formula (2.27) and (2.28), ensures that these changes do not affect the approximation process. 
We observe that the constraint

$$
\forall i \in I: \quad \sum_{j} \sum_{h} j_{h}\left(\mu_{i-e_{h / n}, j}-\mu_{i, j}\right)=0
$$

where $\left(e_{h}\right)_{h=1, \ldots, N}$ denotes the canonical basis of $\mathbb{R}^{N}$, corresponds to the closeness condition, while

$$
\sum_{i, j} j \mu_{i, j}=Q
$$

is the discrete version of the momentum constraint of Mather's measure, which is equivalent to fix the homology class of the measure $\mu$.

Of course, we have $C \neq \emptyset$. Indeed, setting for every $(i, j) \neq(0,0)$

$$
\left\{\begin{array}{l}
\mu_{i, e_{h}}=\frac{1}{|I|} \max \left(Q_{h}, 0\right), \\
\mu_{i,-e_{h}}=\frac{1}{|I|} \max \left(-Q_{h}, 0\right), \\
\mu_{i, j}=0 \quad \text { for } j \neq \pm e_{h}
\end{array}\right.
$$

and $\mu_{0,0}=1-\sum_{h=1}^{N}\left|Q_{h}\right|$, it results $\mu=\sum_{i, j} \mu_{i, j} \delta_{i, j} \in C$.

Lemma 2.2. For every $n \geq 1 \mathbb{P}_{n}$ admits minimizers.

Proof. Let $\mu_{k}$ be a minimizing sequence. Since $C \neq \emptyset$, we have $\mathbb{P}_{n}\left(\mu_{k}\right)<+\infty$ and we may assume that $\mu_{k}$ are atomic measures. It follows that the sequence $\left|\mu_{k}\right|$ is uniformly bounded. Therefore, by weak-star compactness of measures, there exists a convergent subsequence to a measure $\mu$ having compact support. Since $C$ is a closed convex subset of a finite dimensional simplex, it results $\mu \in C$. Since the point of the discretization are fixed, it turns out that $\mathbb{P}_{n}$ is continuous along $\mu_{k}$. Therefore, $\mu$ is a minimum point for $\mathbb{P}_{n}$.

\subsection{Approximation}

The main result of this section is Theorem 2.2 which allow to recover a Mather minimizing measure by solving finite dimensional minimization problems. We state the following approximation result

Theorem 2.1. $\mathbb{P}_{n} \stackrel{\Gamma}{\rightarrow} \mathbb{P}$ with respect to the $\rightarrow$ convergence.

We refer to [16] for an introduction to the $\Gamma$-convergence. To simplify reading, we divide the proof of the above theorem in two lemmas.

Lemma 2.3. Let $\mu \in \mathcal{M}_{c}(T M)$. Then, for every sequence $\mu_{n} \in \mathcal{M}_{c}(T M)$ we have

$$
\mu_{n} \rightarrow \mu \Rightarrow \mathbb{P}(\mu) \leq \liminf _{n \rightarrow+\infty} \mathbb{P}_{n}\left(\mu_{n}\right)
$$

Proof. Suppose that $\liminf _{n \rightarrow+\infty} \mathbb{P}_{n}\left(\mu_{n}\right)<+\infty$. Hence, we may assume that $\mu_{n} \in C$. To simplify notation we denote $\mu_{i, j}^{n}:=\left(\mu_{n}\right)_{i, j}$. If there are infinitely many $\mu_{0,0}^{n}<0$, the penalization term $\Phi_{n}$ forces $\left(\mu_{0,0}^{n}\right)^{-} \rightarrow 0$ as $n \rightarrow+\infty$. Therefore, we get $\left|\mu_{n}\right| \stackrel{*}{\rightarrow} \mu$. This implies $\mu \geq 0$. Since $\left(\mu_{0,0}^{n}\right)^{-} \rightarrow 0$ as $n \rightarrow+\infty$, it follows that actually $\mu$ is a probability measure. Indeed, by weak-star convergence $\mu(T M) \leq 1$. On the other hand, fixed a large compact set $\mathcal{K}$ such that $\operatorname{supp}(\mu) \subset \mathcal{K}, \mu(\partial \mathcal{K})=0$ and $|v| \geq 1$ for every $(x, v) \in \mathcal{K}^{c}$ we get

$$
0 \leq \mu_{n}\left(\mathcal{K}^{c}\right) \leq \int_{\mathcal{K}^{c}}|v| \mathrm{d} \mu_{n}=\int_{\mathcal{K}^{c}}|v| \mathrm{d}\left(\mu_{n}-\mu\right)=\int_{T M}|v| \mathrm{d}\left(\mu_{n}-\mu\right)-\int_{\mathcal{K}}|v| \mathrm{d}\left(\mu_{n}-\mu\right) .
$$

Since $\mu_{n} \rightarrow \mu$ it results $\mu_{n}\left(\mathcal{K}^{c}\right) \rightarrow 0$ as $n \rightarrow+\infty$. Therefore

$$
\mu(T M)=\mu(\mathcal{K}) \geq \limsup _{n \rightarrow+\infty}\left|\mu_{n}\right|(\mathcal{K})=\limsup _{n \rightarrow+\infty}\left(1-\mu_{n}\left(\mathcal{K}^{c}\right)+2\left(\mu_{0,0}^{n}\right)^{-}\right)=1 .
$$


Since $\mu_{n} \rightarrow \mu$ and $\left(\mu_{0,0}^{n}\right)^{-} \rightarrow 0$, Lemma 2.1 still holds and then we deduce that the measure $\mu$ verifies the momentum constraint. It remains to test the closeness constraint. If we denote $D_{h}^{n} \phi(x)=\frac{\phi\left(x+e_{h} / n\right)-\phi(x)}{1 / n}$, by changing variables, the constraint (2.5) for $\mu_{n}$ is equivalent to

$$
\forall \phi \in \mathcal{C}^{1}(M): n \sum_{i} \phi(i) \sum_{h=1}^{N} j_{h}\left(\mu_{i-e_{h} / n, j}^{n}-\mu_{i, j}^{n}\right)=\sum_{i, j} \sum_{h=1}^{N} j_{h} D_{h}^{n} \phi(i) \mu_{i, j}^{n}=\int_{T M}\left\langle v, D^{n} \phi(x)\right\rangle \mathrm{d} \mu_{n}=0,
$$

where $D^{n} \phi(x)=\left(D_{1}^{n} \phi(x), \ldots, D_{N}^{n} \phi(x)\right)$. We evaluate

$$
\begin{aligned}
0=\int_{T M}\left\langle v, D^{n} \phi(x)\right\rangle \mathrm{d} \mu_{n} & =\int_{T M}\langle v, D \phi(x)\rangle \mathrm{d} \mu_{n}-\int_{T M}\left\langle v, D \phi(x)-D^{n} \phi(x)\right\rangle \mathrm{d} \mu_{n} \\
& \leq \int_{T M}\langle v, D \phi(x)\rangle \mathrm{d} \mu_{n}+\left\|D \phi-D^{n} \phi\right\|_{\infty} \int_{T M}|v| \mathrm{d} \mu_{n} .
\end{aligned}
$$

Passing to the limit as $n \rightarrow+\infty$, because of Lemma 2.1, we obtain

$$
\int_{T M}\langle v, D \phi\rangle \mathrm{d} \mu \geq 0
$$

By the arbitrariness of $\phi$ it follows that $\mu$ is a closed measure. Finally, since $L$ is superlinear, without loss of generality we may assume that $L$ is positive on $\mathcal{K}^{c}$. Recalling that $L(0,0)=0$, by the weak-star convergence of measures we obtain

$$
\begin{aligned}
\mathbb{P}(\mu)= & \int_{T M} L(x, v) \mathrm{d} \mu=\int_{\mathcal{K}} L(x, v) \mathrm{d} \mu=\lim _{n \rightarrow+\infty} \int_{\mathcal{K}} L(x, v) \mathrm{d} \mu_{n} \\
= & \lim _{n \rightarrow+\infty}\left(\int_{T M} L(x, v) \mathrm{d} \mu_{n}-\int_{\mathcal{K}^{c}} L(x, v) \mathrm{d} \mu_{n}\right. \\
& \left.+\sum_{i,|j|=n}|j| \mu_{i, j}^{n}-\sum_{i,|j|=n}|j| \mu_{i, j}^{n}+O(n)\left(\mu_{0,0}^{n}\right)^{-}-O(n)\left(\mu_{0,0}^{n}\right)^{-}\right) \\
= & \lim _{n \rightarrow+\infty}\left(\mathbb{P}_{n}\left(\mu_{n}\right)-\int_{\mathcal{K}^{c} \backslash j \mid=n} L(x, v) \mathrm{d} \mu_{n}-O(n)\left(\mu_{0,0}^{n}\right)^{-}-\sum_{i,|j|=n}|j| \mu_{i, j}^{n}\right) \leq \liminf _{n \rightarrow+\infty} \mathbb{P}_{n}\left(\mu_{n}\right) .
\end{aligned}
$$

Lemma 2.4. For every $\mu \in \mathcal{M}_{c}(T M)$ there exists a sequence $\mu_{n} \in \mathcal{M}_{c}(T M)$ such that $\mu_{n} \rightarrow \mu$ and satisfying $\mathbb{P}(\mu)=\lim _{n \rightarrow+\infty} \mathbb{P}_{n}\left(\mu_{n}\right)$.

Proof. We may assume that $\mathbb{P}(\mu)<+\infty$, namely that $\mu$ satisfy the constraints of Mather problem. For every $(i, j) \in I \times J$ consider disjoint squares $Q_{i, j}$ with center in the points $(i, j)$ and size $1 / n$, in such a way, for large $n$, they cover the $\operatorname{supp}(\mu)$. We set $\mu_{i, j}=\mu\left(Q_{i, j}\right)$ and $\mu_{n}=\sum_{i, j} \mu_{i, j} \delta_{i, j}$. We claim that $\int_{T M} f(x, v) \mathrm{d} \mu_{n} \rightarrow \int_{T M} f(x, v) \mathrm{d} \mu$ as $n \rightarrow+\infty$ for every uniformly continuous function $f$. Indeed

$$
\begin{aligned}
\left|\int_{T M} f(x, v) \mathrm{d} \mu-\int_{T M} f(x, v) \mathrm{d} \mu_{n}\right| & =\left|\sum_{i, j} \int_{Q_{i, j}} f(x, v) \mathrm{d} \mu-\sum_{i, j} f(i, j) \mu_{i, j}\right| \\
& =\left|\sum_{i, j} \int_{Q_{i, j}}(f(x, v)-f(i, j)) \mathrm{d} \mu\right| \leq \sum_{i, j} \int_{Q_{i, j}}|f(x, v)-f(i, j)| \mathrm{d} \mu \rightarrow 0
\end{aligned}
$$


as $n \rightarrow+\infty$ since $f$ is uniformly continuous and $\mu$ is a probability measure. Therefore, it also results $\mu_{n} \rightarrow \mu$. Although $\mu_{n}$ are probability measures, in general $\mu_{n}$ does not belong to the constraints set $C$. Therefore, we have to modify this construction. With respect to the closeness constraint, we look for coefficients $\alpha_{i, j} \geq 0$ such that the discrete measure $\mu_{n}^{\prime}$ defined by $\left(\mu_{n}^{\prime}\right)_{i, j}=\mu_{i, j}+\alpha_{i, j}$ satisfies the following conditions

$$
\begin{gathered}
\forall i \in I: \sum_{j} \sum_{h=1}^{N} j_{h}\left(\mu_{i-e_{h / n}, j}^{\prime}-\mu_{i, j}^{\prime}\right)=0 \\
\left|\mu_{n}-\mu_{n}^{\prime}\right| \rightarrow 0 .
\end{gathered}
$$

For every $i \in I$, condition (2.8) is equivalent to

$$
\sum_{j} \sum_{h=1}^{N} j_{h}\left(\alpha_{i-e_{h / n}, j}-\alpha_{i, j}\right)=c_{i}:=\sum_{j} \sum_{h=1}^{N} j_{h}\left(\mu_{i, j}-\mu_{i-e_{h / n}, j}\right),
$$

while condition $(2.9)$ is equivalent to

$$
\sum_{i, j} \alpha_{i, j} \rightarrow 0
$$

Setting $c=\left(c_{i}\right)_{i \in I}, z=\mu_{i, j}, \alpha=\alpha_{i, j}$, condition (2.10) can be written as

$$
B \alpha=c,
$$

for a suitable matrix $B$. We search solutions $\alpha$ of (2.12) supported on $\operatorname{supp}(\mu)$, i.e. $\alpha_{i, j}=0$ whenever $|j|>R$ for a suitable large $R>0$. By definition of the coefficients $c_{i},-z$ is a such solution of (2.12). Therefore, all the solutions of (2.12) are of the form $\beta-z$ with $\beta \in \operatorname{ker}(B)$. Since the vectors whose components are all equal belong to $\operatorname{ker}(B)$, we can find positive solutions of (2.12). Denoting by $\mathbb{I}$ the vector whose components are all equal to 1 , we take $\alpha$ as a solution of the following minimization problem

$$
\left\{\begin{array}{l}
\min \langle\alpha, \mathbb{I}\rangle \\
\alpha \geq 0 \\
B \alpha=c
\end{array}\right.
$$

By duality we also have a solution $y=\left(y_{i}\right)_{i \in I}$ of the problem

$$
\left\{\begin{array}{l}
\max \langle c, y\rangle \\
B^{t} y \leq \mathbb{I}
\end{array}\right.
$$

We use duality to compute $B^{t}$ as follows

$$
\left\langle B^{t} y, x\right\rangle=\langle y, B x\rangle=\sum_{i} y_{i}\left(\sum_{j} \sum_{h=1}^{N} j_{h}\left(x_{i-e_{h / n}, j}-x_{i, j}\right)\right)=\sum_{i, j} \sum_{h=1}^{N} j_{h}\left(y_{i+e_{h / n}}-y_{i}\right) x_{i, j} .
$$

Therefore, the $(i, j)$ th component of $B^{t} y$ is

$$
\sum_{h=1}^{N} j_{h}\left(y_{i+e_{h / n}}-y_{i}\right)
$$

Hence, the constraint of problem (2.14) yields

$$
\forall(i, j) \in I \times J: \sum_{h=1}^{N} j_{h}\left(y_{i+e_{h / n}}-y_{i}\right) \leq 1 .
$$


Choosing $j= \pm e_{h}$ in the above formula we obtain

$$
\forall i \in I, \forall h=1, \ldots, N:\left|y_{i+e_{h / n}}-y_{i}\right| \leq 1
$$

By the slackness condition we have

$$
\begin{aligned}
\sum_{i, j} \alpha_{i, j}=\langle\alpha, \mathbb{I}\rangle & =\langle c, y\rangle=\sum_{i} y_{i} c_{i}=\sum_{i} y_{i}\left(\sum_{j} \sum_{h=1}^{N} j_{h}\left(\mu_{i, j}-\mu_{i-e_{h / n}, j}\right)\right) \\
& =\sum_{i, j} \sum_{h=1}^{N} j_{h}\left(y_{i}-y_{i+e_{h / n}}\right) \mu_{i, j} .
\end{aligned}
$$

In particular, the above computation shows that $\sum_{i} c_{i}=0$ (choose $y_{i}=$ constant). Therefore, by adding a constant, we may assume that $y_{i}=0$ for some $i \in I$. We have to prove that actually $\sum_{i} y_{i} c_{i} \rightarrow 0$. First, because of (2.15), it results that the function $\phi_{n}(i)=y_{i}$ is a Lipschitz function on the discrete grid $I$. Therefore, by standard extension theorems for Lipschitz functions, we obtain a Lipschitz function $\phi_{n}$ on the whole flat torus $\mathbb{T}^{N}$ whose Lipschitz constant grows as the discrete step $n$, i.e. $\operatorname{Lip}\left(\phi_{n}\right) \approx n$. Therefore, the sequence of functions $\varphi_{n}=\frac{1}{n} \phi_{n}$ is equi-Lipschitz and equi-bounded. By the Ascoli-Arzelà theorem, by passing to a subsequence, we find a Lipschitz function $\varphi$ such that $\left\|\varphi_{n}-\varphi\right\|_{\infty} \rightarrow 0$. On the other hand, since $\mu$ is a closed measure and $\mu_{n} \rightarrow \mu$, with the same notation of the proof of Lemma 2.3 , for every $\phi \in \mathcal{C}^{1}$ we get

$$
\begin{aligned}
-n \sum_{i} \phi(i) c_{i} & =\sum_{i, j} \sum_{h=1}^{N} n \phi(i) j_{h}\left(\mu_{i-e_{h / n}, j}-\mu_{i, j}\right)=\sum_{i, j} \sum_{h=1}^{N} j_{h} D_{h}^{n} \phi(i) \mu_{i, j}^{n}=\int_{T M}\left\langle v, D^{n} \phi(x)\right\rangle \mathrm{d} \mu_{n} \\
& \leq\left\|D \phi-D^{n} \phi\right\|_{\infty} \int_{T M}|v| \mathrm{d} \mu_{n}+\int_{T M}\langle D \phi(x), v\rangle \mathrm{d} \mu_{n} \rightarrow 0
\end{aligned}
$$

as $n \rightarrow+\infty$. Consider the linear operator on the space $\left(\operatorname{Lip}(M),\|\cdot\|_{\infty}\right)$ of Lipschitz functions defined by $\mathcal{L}_{n}(f):=n \sum_{i} f(i) c_{i}$. By the uniform boundedness principle on the space of $\mathcal{C}^{1}$ functions, taking into account the computation (2.17), for every $f \in \mathcal{C}^{1}$ it results

$$
\left|\mathcal{L}_{n}(f)\right| \leq K\left(\|f\|_{\infty}+\operatorname{Lip}(f)\right)
$$

If $f$ is a Lipschitz function, by standard approximation results (using for instance convolution kernels) we find $f_{n} \in \mathcal{C}^{1}$ such that $\left\|f-f_{n}\right\|_{\infty} \leq 1 / n$ and $\operatorname{Lip}\left(f_{n}\right) \leq \operatorname{Lip}(f)$. Since $\mu \in \mathcal{P}_{c}(T M)$, it follows $\sum_{i}\left|c_{i}\right| \leq M$. Hence we compute

$$
\left|\mathcal{L}_{n}(f)\right| \leq\left|\mathcal{L}_{n}\left(f-f_{n}\right)\right|+\left|\mathcal{L}_{n}\left(f_{n}\right)\right| \leq n\left\|f-f_{n}\right\|_{\infty} \sum_{i}\left|c_{i}\right|+K\left(\|f\|_{\infty}+\operatorname{Lip}(f)\right) \leq C(f)
$$

Applying again the Banach-Steinhaus theorem we find

$$
n \sum_{i}\left|c_{i}\right|=\left\|\mathcal{L}_{n}\right\| \leq C
$$

If $f \in \operatorname{Lip}(M)$, for every $\phi \in \mathcal{C}^{1}$ we have the estimate

$$
\left|n \sum_{i} f(i) c_{i}\right| \leq n \sum_{i}\left|c_{i}\right|\|f-\phi\|_{\infty}+\left|n \sum_{i} \phi(i) c_{i}\right| \leq C\|f-\phi\|_{\infty}+\left|n \sum_{i} \phi(i) c_{i}\right| .
$$


Using the boundedness condition (2.18), by (2.17) and by density of $\mathcal{C}^{1}$ functions in $\operatorname{Lip}(M)$, for every Lipschitz function $f$ it results

$$
n \sum_{i} f(i) c_{i} \rightarrow 0
$$

as $n \rightarrow+\infty$. Now we come back to the estimation of the coefficient $\alpha_{i, j}$. By (2.16) we have

$$
\sum_{i, j} \alpha_{i, j}=\sum_{i} y_{i} c_{i}=n \sum_{i} \varphi_{n}(i) c_{i} \leq n\left\|\varphi-\varphi_{n}\right\|_{\infty} \sum_{i}\left|c_{i}\right|+n \sum_{i} \varphi(i) c_{i} \leq C\left\|\varphi-\varphi_{n}\right\|_{\infty}+n \sum_{i} \varphi(i) c_{i} \rightarrow 0
$$

as $n \rightarrow+\infty$. Therefore, condition (2.11) holds. Without loss of generality, normalizing we may assume that $\mu_{n}^{\prime}(T M)=1$. At this point we need another modification to satisfy the momentum constraint. To this aim we look for coefficients $s_{i, j} \geq 0$ such that the discrete measure $\bar{\mu}_{n}$ defined by $\left(\bar{\mu}_{n}\right)_{i, j}=\mu_{i, j}^{\prime}+s_{i, j}$ has the right momentum. For $h=1, \ldots, N$, denoting by

$$
\begin{aligned}
& R_{h}=\sum_{i, j} j_{h} \mu_{i, j} \\
& R_{h}^{\prime}=\sum_{i, j} j_{h} \mu_{i, j}^{\prime} \\
& s_{h}=\frac{Q_{h}-R_{h}^{\prime}}{n|I|},
\end{aligned}
$$

for every $(i, j) \in I \times J$, we set

$$
\left\{\begin{array}{l}
s_{i, n e_{h}}=\max \left(s_{h}, 0\right) \\
s_{i,-n e_{h}}=\max \left(-s_{h}, 0\right) \\
s_{i, j}=0 \text { for } j \neq \pm n e_{h}
\end{array}\right.
$$

Therefore, the momentum of $\bar{\mu}_{n}$ is given by

$$
\sum_{i, j} j_{h}\left(\bar{\mu}_{n}\right)_{i, j}=R_{h}^{\prime}+\sum_{i, j} j_{h} s_{i, j}=R_{h}^{\prime}+n|I| s_{h}=R_{h}^{\prime}+Q_{h}-R_{h}^{\prime}=Q_{h}
$$

Observe that the choice of $s_{i, j}$ does not modify the closeness constraint. Indeed, for every $i \in I$ we have

$$
\begin{aligned}
\sum_{j} \sum_{h=1}^{N} j_{h}\left(\left(\bar{\mu}_{n}\right)_{i-e_{h / n}, j}-\left(\bar{\mu}_{n}\right)_{i, j}\right)= \pm n & \sum_{j= \pm n e_{h}} \sum_{h=1}^{N}\left(\mu_{i-e_{h / n}, j}^{\prime}+s_{h}-\mu_{i, j}^{\prime}-s_{h}\right) \\
& +\sum_{j \neq \pm n e_{h}} \sum_{h=1}^{N} j_{h}\left(\mu_{i-e_{h / n}, j}^{\prime}-\mu_{i, j}^{\prime}\right)=\sum_{j} \sum_{h=1}^{N} j_{h}\left(\mu_{i-e_{h / n}, j}^{\prime}-\mu_{i, j}^{\prime}\right)=0 .
\end{aligned}
$$

Since

$$
\left|Q_{h}-R_{h}\right|=\left|\sum_{i, j} \int_{Q_{i, j}}\left(v_{h}-j_{h}\right) \mathrm{d} \mu\right| \leq \frac{K}{n}
$$


for a constant $K>0$ which depends only on the dimension $N$ of the space, we can evaluate

$$
\begin{aligned}
\sum_{i, j} s_{i, j}=|I| \sum_{h=1}^{N}\left|s_{h}\right| & =\frac{1}{n} \sum_{h=1}^{N}\left|Q_{h}-R_{h}^{\prime}\right| \leq \frac{1}{n} \sum_{h=1}^{N}\left|Q_{h}-R_{h}\right|+\frac{1}{n} \sum_{i, j}|j| \alpha_{i, j} \\
& \leq \frac{N K}{n^{2}}+\frac{1}{n} \sum_{i, j}|j| \alpha_{i, j} \rightarrow 0, \\
\sum_{i, j}|j| s_{i, j} & =n \sum_{i, j} s_{i, j} \leq \frac{N K}{n}+\sum_{i, j}|j| \alpha_{i, j} \rightarrow 0,
\end{aligned}
$$

as $n \rightarrow+\infty$ because of (2.11) and since $\alpha$ has compact support. Moreover, we estimate

$$
\left|\bar{\mu}_{n}-\mu_{n}\right|=\sum_{i, j}\left|\left(\bar{\mu}_{n}\right)_{i, j}-\mu_{i, j}\right| \leq \sum_{i, j} \alpha_{i, j}+\sum_{i, j} s_{i, j} .
$$

By (2.11) and (2.24) we infer

$$
\lim _{n \rightarrow+\infty}\left|\mu_{n}-\bar{\mu}_{n}\right|=0 .
$$

It remains to satisfy the probability constraint. This can be done by subtracting the mass needed at the origin, without changing neither the momentum or the closeness constraint. More precisely, since

$$
\sum_{i, j}\left(\bar{\mu}_{n}\right)_{i, j}=\sum_{i, j} \mu_{i, j}^{\prime}+\sum_{i, j} s_{i, j}=1+\sum_{i, j} s_{i, j}
$$

if we put $\left(\bar{\mu}_{n}\right)_{0,0}=\mu_{0,0}^{\prime}-\sum_{i, j} s_{i, j}$ we also have $\sum_{i, j}\left(\bar{\mu}_{n}\right)_{i, j}=1$. Condition (2.26) still holds since by $(2.24)$ we have added infinitesimal quantities. At this point it is easy to verify the weak-star convergence. Indeed, if $f \in \mathcal{C}_{c}(T M)$ we have

$$
\begin{aligned}
\left|\int_{T M} f(x, v) \mathrm{d} \mu-\int_{T M} f(x, v) \mathrm{d} \bar{\mu}_{n}\right| & \leq\left|\int_{T M} f(x, v) \mathrm{d} \mu-\int_{T M} f(x, v) \mathrm{d} \mu_{n}\right|+\left|\int_{T M} f(x, v) \mathrm{d} \mu_{n}-\int_{T M} f(x, v) \mathrm{d} \bar{\mu}_{n}\right| \\
& \leq\left|\int_{T M} f(x, v) \mathrm{d} \mu-\int_{T M} f(x, v) \mathrm{d} \mu_{n}\right|+\|f\|_{\infty}\left|\bar{\mu}_{n}-\mu_{n}\right| \rightarrow 0 .
\end{aligned}
$$

In order to verify the convergence $\bar{\mu}_{n} \rightarrow \mu$, we need to check the convergence of momentum. By (2.9) and (2.25) we get

$$
\left|\int_{T M}\right| v\left|\mathrm{~d} \mu-\int_{T M}\right| v\left|\mathrm{~d} \bar{\mu}_{n}\right| \leq\left|\int_{T M}\right| v\left|\mathrm{~d} \mu-\int_{T M}\right| v\left|\mathrm{~d} \mu_{n}^{\prime}\right|+\sum_{i, j}|j| s_{i, j} \rightarrow 0 .
$$

Finally, recalling that $L(0,0)=0$, we evaluate

$$
\begin{aligned}
\left|\mathbb{P}(\mu)-\mathbb{P}_{n}\left(\bar{\mu}_{n}\right)\right| & =\left|\int_{T M} L(x, v) \mathrm{d} \mu-\int_{T M} L(x, v) \mathrm{d} \bar{\mu}_{n}-\Phi_{n}\left(\bar{\mu}_{n}\right)\right| \\
& \leq\left|\int_{T M} L(x, v) \mathrm{d} \mu-\sum_{i, j} L(i, j) \mu_{i, j}^{\prime}\right|+\sum_{i,|j|=n}|j| s_{i, j}+O(n)\left(\left(\bar{\mu}_{n}\right)_{0,0}\right)^{-} .
\end{aligned}
$$


Using (2.9), (2.24) and (2.25) it follows

$$
\begin{aligned}
\left|\mathbb{P}(\mu)-\mathbb{P}_{n}\left(\bar{\mu}_{n}\right)\right| & \leq\left|\int_{T M} L(x, v) \mathrm{d} \mu-\sum_{i, j} L(i, j) \mu_{i, j}^{\prime}\right|+\sum_{i,|j|=n}|j| s_{i, j}+O(n)\left(\left(\bar{\mu}_{n}\right)_{0,0}\right)^{-} \\
& =\left|\int_{T M} L(x, v) \mathrm{d} \mu-\sum_{i, j} L(i, j) \mu_{i, j}^{\prime}\right|+\sum_{i,|j|=n}|j| s_{i, j}+O(n) \sum_{i, j} s_{i, j} \rightarrow 0 .
\end{aligned}
$$

Theorem 2.2. Let $\mu_{n}$ be minimum points of $\mathbb{P}_{n}$. Then there exists a sequence of discrete measures $\bar{\mu}_{n}$ and a Mather minimizing measure $\mu$ such that $\bar{\mu}_{n} \rightarrow \mu$. Moreover, by passing to a subsequence, it results

$$
\mathbb{P}(\mu)=\lim _{n \rightarrow+\infty} \mathbb{P}_{n}\left(\mu_{n}\right) .
$$

Proof. Let $K=\max \{L(x, v):(x, v) \in T M,\|v\|=1\}$. Without loss of generality we may assume that $0 \leq K \leq 1 / \sqrt{N}$. By superlinearity, there exists $k>1$ such that for every $x \in M$ it results $L(x, v)>\sqrt{N} K|v|$ whenever $|v|>k$. For every $(i, j) \in I \times J$ we set

$$
\left\{\begin{array}{l}
\left(\bar{\mu}_{n}\right)_{i, e_{h}}=\left(\mu_{n}\right)_{i, e_{h}}+\sum_{|j|>k} \max \left(j_{h}\left(\mu_{n}\right)_{i, j}, 0\right) \\
\left(\bar{\mu}_{n}\right)_{i,-e_{h}}=\left(\mu_{n}\right)_{i,-e_{h}}+\sum_{|j|>k} \max \left(-j_{h}\left(\mu_{n}\right)_{i, j}, 0\right) \\
\left(\bar{\mu}_{n}\right)_{i, j}=\left(\mu_{n}\right)_{i, j} \quad \text { for } j \neq \pm e_{h}, 0<|j| \leq k \\
\left(\bar{\mu}_{n}\right)_{i, j}=0 \quad \text { for }|j|>k \text { or }|j|=0 .
\end{array}\right.
$$

By the above modification it results that the new discrete measures $\bar{\mu}_{n}$ is positive in $T M$ and, since we may assume $\mu_{n} \in C$, they still satisfy the momentum and closeness constraints (2.6) and (2.5). We need at most to check the probability constraint. Let $M=\sum_{i, j}\left(\bar{\mu}_{n}\right)_{i, j}$. If $M<1$, we add the needed mass at the origin. If $M>1$ we normalize by considering the measure $\bar{\mu}_{n} / M$. This normalized measure is still closed but the momentum amounts to $\frac{Q}{M}$. Following the construction performed in the proof of Lemma 2.4, we can obtain the right momentum by adding the quantities

$$
s_{h}=\frac{Q_{h}-Q_{h} / M}{n|I|}
$$

as done in (2.22). It remains to subtract the mass in excess, which is an infinitesimal quantity, at the origin. Setting $\varepsilon_{n}=O(n) \sum_{i, j} s_{i, j}$, because of (2.24) it results $\varepsilon_{n} \rightarrow 0$ as $n \rightarrow+\infty$. In any case, for every $\nu \in \mathcal{M}_{c}(T M)$ we get

$$
\begin{aligned}
\mathbb{P}_{n}\left(\bar{\mu}_{n}\right) & \leq \sum_{i,|j| \leq k} L(i, j)\left(\mu_{n}\right)_{i, j}+\sum_{i,|j|>k} \sum_{h=1}^{N} L\left(i, \pm e_{h}\right)\left|j_{h}\right|\left(\mu_{n}\right)_{i, j}+\varepsilon_{n} \\
& \leq \sum_{i,|j| \leq k} L(i, j)\left(\mu_{n}\right)_{i, j}+\sqrt{N} K \sum_{i,|j|>k}|j|\left(\mu_{n}\right)_{i, j}+\varepsilon_{n}<\mathbb{P}_{n}\left(\mu_{n}\right)+\varepsilon_{n} \leq \mathbb{P}_{n}(\nu)+\varepsilon_{n} .
\end{aligned}
$$

Therefore, $\bar{\mu}_{n}$ is an $\varepsilon_{n}$-minimizer for $\mathbb{P}_{n}$. Furthermore, since $\left|\bar{\mu}_{n}\right|$ is equibounded and the supports are contained in a same large compact set, by passing to a convergent subsequence we have $\bar{\mu}_{n} \rightarrow \mu$. Moreover, for every $\nu \in \mathcal{M}_{c}(T M)$, by Lemma 2.4 we find a sequence $\nu_{n} \in \mathcal{M}_{c}(T M)$ such that $\mathbb{P}_{n}\left(\nu_{n}\right) \rightarrow \mathbb{P}(\nu)$ as $n \rightarrow+\infty$. By $(2.30)$ we get $\mathbb{P}_{n}\left(\bar{\mu}_{n}\right) \leq \mathbb{P}_{n}\left(\nu_{n}\right)+\varepsilon_{n}$. Letting $n \rightarrow+\infty$, by Lemma 2.3 we infer

$$
\mathbb{P}(\mu) \leq \liminf _{n \rightarrow+\infty} \mathbb{P}_{n}\left(\bar{\mu}_{n}\right) \leq \lim _{n \rightarrow+\infty} \mathbb{P}_{n}\left(\nu_{n}\right)=\mathbb{P}(\nu) .
$$


Therefore $\mu$ is a Mather minimizing measure. Moreover, again by Lemma 2.4, we find a sequence $\mu_{n}^{\prime}$ such that $\mathbb{P}_{n}\left(\mu_{n}^{\prime}\right) \rightarrow \mathbb{P}(\mu)$ as $n \rightarrow+\infty$. Since $\mu_{n}$ are minimum points of $\mathbb{P}_{n}$ we have

$$
\limsup _{n \rightarrow+\infty} \mathbb{P}_{n}\left(\mu_{n}\right) \leq \lim _{n \rightarrow+\infty} \mathbb{P}_{n}\left(\mu_{n}^{\prime}\right)=\mathbb{P}(\mu) .
$$

On the other hand, by (2.30) we get

$$
\mathbb{P}(\mu) \leq \liminf _{n \rightarrow+\infty} \mathbb{P}_{n}\left(\bar{\mu}_{n}\right) \leq \liminf _{n \rightarrow+\infty} \mathbb{P}_{n}\left(\mu_{n}\right)
$$

\section{LiNEAR PROGRAMMING INTERPRETATION}

The discrete problems of minimizing $\mathbb{P}_{n}$ can be reformulate into a linear programming framework. Indeed, splitting $\mu_{0,0}=\mu_{0,0}^{+}-\mu_{0,0}^{-}$, therefore increasing the dimension of the problem, we set

$$
\begin{aligned}
& \lambda_{i, j}=L(i, j) \quad|j| \neq n \\
& \lambda_{0,0}^{+}=0, \quad \lambda_{0,0}^{-}=O(n) \\
& \lambda_{i, j}=|j| \quad \text { for every }|j|=n .
\end{aligned}
$$

Therefore, minimize the functional $\mathbb{P}_{n}$ is equivalent to

$$
\operatorname{minimize}\left(\sum_{i, j} \lambda_{i, j} \mu_{i, j}\right)
$$

subject to the following constraints

$$
\begin{aligned}
& \mu_{i, j}, \mu_{0,0}^{+}, \mu_{0,0}^{-} \geq 0 \\
& \forall i \in I: \sum_{h=1}^{N} \sum_{j} j_{h}\left(\mu_{i-e_{h} / n, j}-\mu_{i, j}\right)=0 \\
& \sum_{i, j} \mu_{i, j}+\mu_{0,0}^{+}-\mu_{0,0}^{-}=1 \\
& \sum_{i, j} j \mu_{i, j}=Q .
\end{aligned}
$$

In this problem we have $h=|I|+N+1$ equality constraints for $k=|I||J|+1$ unknowns. Denoting respectively by $\mu$ and $\lambda$ the vectors of $k$ unknown components $\mu_{i, j}$ and $\lambda_{i, j}$, and the vector

$$
b=(\underbrace{0, \ldots, 0}_{|I| \text { zeros }}, 1, Q_{1}, \ldots, Q_{N}),
$$

the above problem can be written in the form

$$
\left\{\begin{array}{l}
\operatorname{minimize}\langle\lambda, \mu\rangle \\
\mu \geq 0 \\
A \mu=b
\end{array}\right.
$$

for a suitable $h \times k$ matrix $A$. As standard in linear programming, problem (3.2) admits the dual formulation

$$
\left\{\begin{array}{l}
\operatorname{maximize}\langle y, b\rangle \\
A^{t} y \leq \lambda
\end{array}\right.
$$

where $A^{t}$ is the transpose matrix of $A$. 


\subsection{The infinite dimensional dual problem}

In [9] it is proposed a linear programming interpretation of Mather's problem, while a linear programming interpretation is a well known fact for the Monge-Kantorovich problem (see for example [7]). In particular, for $\mu \in \mathcal{M}_{c}(T M)$ define:

and consider the continuous linear operator

$$
\phi \in \mathcal{C}^{1}(M) \mapsto L_{\mu}(\phi)=\int_{T M}\langle D \phi(x), v\rangle \mathrm{d} \mu,
$$

$$
A: \mathcal{M}_{c}(T M) \rightarrow \mathcal{C}^{1}(M)^{*} \times \mathbb{R} \times \mathbb{R}^{N}
$$

defined by

$$
A \mu=\left(L_{\mu}, \mu(T M), \int_{T M} v \mathrm{~d} \mu\right) .
$$

If we denote by $c^{*}$, the linear functional associated to $L$ by $\mu \mapsto \int_{T M} L(x, v) \mathrm{d} \mu$, fixed $Q \in \mathbb{R}^{N}$ and setting $(0,1, Q)=b$, Mather's problem can be reformulated as the following infinite dimensional linear programming problem

$$
\left\{\begin{array}{l}
\min \left\langle c^{*}, \mu\right\rangle \\
\mu \geq 0 \\
A \mu=b
\end{array}\right.
$$

The dual formulation of problem (3.4) amounts to

$$
\left\{\begin{array}{l}
\max \left\langle y^{*}, b\right\rangle \\
A^{*} y^{*} \leq c^{*}
\end{array}\right.
$$

However, for infinite dimensional linear programming problems, the dual problem could not admit solutions despite the existence of solutions of the primal one. Moreover, since the space $\mathcal{C}^{1}(M)$ is not reflexive, to a solution $y^{*}$ of the dual problem does not correspond in general a $\mathcal{C}^{1}$ function. Actually, in [9] it is shown that there is no duality gap between the two problem and that if we have a solution $y^{*}$ of $(3.5)$ such that $y^{*}=\left(u, \omega_{0}, P\right) \in \mathcal{C}^{1}(M) \times \mathbb{R} \times \mathbb{R}^{N}$ then $u$ solve the Hamilton-Jacobi equation

$$
H\left(x, P+d_{x} u\right)=\bar{H}(P) \quad \text { on } \operatorname{supp}\left(\pi_{x}(\mu)\right),
$$

where $H$ is the Hamiltonian associated to the Lagrangian $L(x, v)$, while $\bar{H}(P)=-\omega_{0}, \mu$ is a solution of (3.4) and $\pi_{x}(\mu)$ is the push-forward of $\mu$ through the projection of $T M$ on $M$. Hence the dual of Mather's problem predict the weak KAM equation (see $[5,8,10,11]$ ). Although in this abstract framework only a weak duality result holds, we can justify a strong duality relation for the Mather's problem by the following considerations. First we recall that Mather's problem can be formulated in terms of merely closed measures. Precisely we have the following (see $[17,19])$ :

Theorem 3.1. For every Mather minimizing measure $\mu$ with $[\mu]=Q$ there exists $P \in \mathbb{R}^{N}$ such that

$$
\mathcal{A}(\mu)+P \cdot Q=-c(L+P)
$$

where $-c(L+P)=\min \left\{\int(L+P) \mathrm{d} \nu \mid \nu \in \mathcal{M}^{c}\right\}$ is the Mañé critical value (see also [5,10,13]) corresponding to the Lagrangian $(L+P)(x, v)=L(x, v)+\langle P, v\rangle$.

We state the following approximation theorem for the dual problem (3.5):

Theorem 3.2. Let $y_{n}$ be solutions of the finite dimensional linear programming problems (3.3). Then there exists a solution $y^{*}$ of problem (3.5) such that, by passing to a subsequence,

$$
\lim _{n \rightarrow+\infty}\left\langle y_{n}, b\right\rangle=\left\langle y^{*}, b\right\rangle \cdot
$$


Proof. Let $\mu_{n}$ be solutions of problems (3.2). By Theorem 2.2, passing to a subsequence, we find a Mather minimizing measure $\mu$ such that $\lim _{n \rightarrow+\infty} \mathbb{P}_{n}\left(\mu_{n}\right)=\mathbb{P}(\mu)$. By Theorem 3.1 we find $P \in \mathbb{R}^{N}$ such that

$$
\mathcal{A}(\mu)+P \cdot Q=-c(L+P) .
$$

Setting $w_{0}=-c(L+P)$ and $w=-P$, as shown in [11] we find $u \in \mathcal{C}^{1}$ such that

$$
H(x, w+D u) \leq-w_{0} \Leftrightarrow w_{0}+v \cdot(w+D u(x)) \leq L(x, v)
$$

Consider $y^{*}=\left(u, w_{0}, w\right)$. We check that such $y^{*}$ satisfies the constraint $A^{*} y^{*} \leq c^{*}$ of the dual problem (3.5). Indeed, it results

$$
\left\langle A^{*} y^{*}, \mu\right\rangle=\left\langle y^{*}, A \mu\right\rangle=\int_{T M}\left(w_{0}+\langle w+D u, v\rangle\right) \mathrm{d} \mu \leq \int_{T M} L(x, v) \mathrm{d} \mu=\left\langle c^{*}, \mu\right\rangle .
$$

Taking in account (3.6), we evaluate

$$
\left\langle y^{*}, b\right\rangle=w_{0}+w \cdot Q=-c(L+P)-P \cdot Q=\mathcal{A}(\mu)=\left\langle c^{*}, \mu\right\rangle
$$

By weak duality, it follows that $y^{*}$ is optimal for the dual problem (3.5). Finally, recalling the discrete approximation for the primal problem and by the finite dimensional duality, we deduce a discrete approximation for the dual problem. In fact, if $y_{n}$ are solutions of the finite dimensional dual problems (3.3), by passing to a subsequence we have

$$
\lim _{n \rightarrow+\infty}\left\langle y_{n}, b\right\rangle=\lim _{n \rightarrow+\infty} \mathbb{P}_{n}\left(\mu_{n}\right)=\mathbb{P}(\mu)=\mathcal{A}(\mu)=\left\langle c^{*}, \mu\right\rangle=\left\langle y^{*}, b\right\rangle
$$

It would be interesting to investigate if also $y_{n} \rightarrow y^{*}$. This question is related to the numerical approximation of the Hamilton-Jacobi equations and to the computing of the effective Hamiltonian. For an account on numerical results linked to these questions we refer to $[12,20]$.

Acknowledgements. The author wishes to thank L. De Pascale and M.S. Gelli for useful discussions on the subject.

\section{REFERENCES}

[1] L. Ambrosio, N. Fusco and D. Pallara, Functions of Bounded Variation and Free Discontinuity Problems. Oxford University Press, New York, USA (2000).

[2] E.J. Anderson and P. Nash, Linear Programming in Infinite Dimensional Spaces. Wiley (1987).

[3] V. Bangert, Minimal measures and minimizing closed normal one-currents. GAFA Geom. Funct. Anal. 9 (1999) $413-427$.

[4] P. Bernard and B. Buffoni, Optimal mass transportation and Mather theory. J. Eur. Math. Soc. (JEMS) 9 (2007) 85-121.

[5] G. Contreras and R. Iturriaga, Global Minimizers of Autonomous Lagrangians. Coloquio Brasileiro de Matematica. IMPA, Rio de Janeiro, Brazil (1999).

[6] L. De Pascale, M.S. Gelli and L. Granieri, Minimal measures, one-dimensional currents and the Monge-Kantorovich problem. Calc. Var. 27 (2006) 1-23.

[7] L.C. Evans, Partial differential equations and Monge-Kantorovich mass transfer, in Current Developments in Mathematics, 1997, S.T. Yau Ed., International Press (1998).

[8] L.C. Evans, Some new PDE methods for weak KAM theory. Calc. Var. Partial Differ. Eq. 17 (2003) 159-177.

[9] L.C. Evans and D. Gomes, Linear programming interpretation of Mather's variational principle. ESAIM: COCV 8 (2002) 693-702.

[10] A. Fathi, The Weak KAM Theorem in Lagrangian Dynamics, Cambridge Studies in Advanced Mathematics 88. Cambridge University Press, Cambridge, UK (2008).

[11] A. Fathi and A. Siconolfi, Existence of $C^{1}$ critical subsolutions of the Hamilton-Jacobi equation. Invent. Math. 155 (2004) 363-388.

[12] D. Gomes and A.M. Oberman, Computing the effective Hamiltonian using a variational approach. SIAM J. Control Optim. 43 (2004) 792-812. 
[13] L. Granieri, Mass Transportation Problems and Minimal Measures. Ph.D. Thesis in Mathematics, Pisa, Italy (2005).

[14] L. Granieri, On action minimizing measures for the Monge-Kantorovich problem. NoDEA 14 (2007) 125-152.

[15] J. Jost, Riemannian Geometry and Geometric Analysis. Springer (2002).

[16] J. Jost and X. Li-Jost, Calculus of Variations, Cambridge Studies in Advanced Mathematics 64. Cambridge University Press, Cambridge, UK (1998).

[17] R. Mañé, Generic properties and problems of minimizing measures of Lagrangian systems. Nonlinearity 9 (1996) 273-310.

[18] J.N. Mather, Minimal measures. Comment. Math. Helv. 64 (1989) 375-394.

[19] J.N. Mather, Action minimizing invariant measures for positive definite Lagrangian systems. Math. Z. 207 (1991) $169-207$.

[20] M. Rorro, An approximation scheme for the effective Hamiltonian and applications. Appl. Numer. Math. 56 (2006) 1238-1254.

[21] S.M. Sinha, Mathematical Programming. Elsevier (2006). 